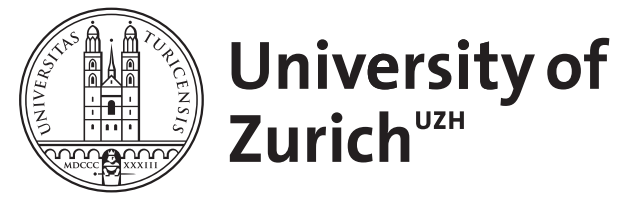
Archive

University of Zurich

University Library

Strickhofstrasse 39

CH-8057 Zurich

www.zora.uzh.ch

Year: 2014

High-Pressure freezing: current state and future prospects

Kaech, Andres ; Ziegler, Urs

DOI: https://doi.org/10.1007/978-1-62703-776-1_8

Posted at the Zurich Open Repository and Archive, University of Zurich

ZORA URL: https://doi.org/10.5167/uzh-91816

Journal Article

Accepted Version

Originally published at:

Kaech, Andres; Ziegler, Urs (2014). High-Pressure freezing: current state and future prospects. Methods in Molecular Biology, 1117:151-171.

DOI: https://doi.org/10.1007/978-1-62703-776-1_8 


\title{
High-Pressure Freezing: Current State and Future Prospects
}

\author{
Andres Kaech and Urs Ziegler
}

\begin{abstract}
In this chapter we discuss the latest developments in the field of high-pressure freezing (HPF). The Leica HPF machine EM HPMI00 is discussed in detail due to significant changes compared to its predecessor model. Its centerpiece is a multipart polymer cartridge which holds the specimen carrier sandwich and guides it automatically through the freezing process until immersed in liquid nitrogen. The cartridge can be adapted to the specimen and carrier geometry to optimize the flow of liquid nitrogen and hence rapid cooling. Dedicated cartridges are available for a variety of different carriers, including carriers for samples of up to $5 \mathrm{~mm}$ in diameter. Cartridge-specific handling and carrier assemblies are described extensively for freezing samples in aluminum specimen carriers, cell cultures grown on Sapphire discs, suspensions for freeze-fracturing, and specimens for cryo-sectioning. Additionally, we include an advanced technique to freeze monolayer cell cultures on Sapphire discs with the Leica EM PACT2 HPF machine using a composite carrier.
\end{abstract}

Key words High-pressure freezing, Freeze-substitution, Freeze-fracturing, Cryo-sectioning, Monolayer cell cultures, Sapphire disc

\section{Introduction}

Cryo-immobilization is the optimum technique to preserve the ultrastructure of biological specimens or more generally, soft condensed matter. However, freezing of samples thicker than a few microns without the use of antifreezing agents and at ambient pressure leads to considerable ice crystal formation and distortion of the biological material. Such samples can only be frozen adequately if intracellular antifreezing agents are used or under high pressure. Intracellular antifreezing agents are normally not incorporated by living cells or organisms under physiological conditions or are problematic concerning normal physiology of the cell. High-pressure freezing (HPF) was developed to overcome these drawbacks and allows adequate freezing of native biological samples of up to $200 \mu \mathrm{m}$ in thickness. The optimum pressure of 
2,100 bar needs to be applied to the sample within a few milliseconds followed by immediate rapid cooling to prevent the introduction of artifacts caused by high pressure (e.g., changes of dissociation constants). HPF became a state-of-the-art technique to contribute to the elucidation of numerous scientific questions which cannot be answered using room temperature preparation techniques like chemical fixation.

Currently, four different HPF machines are available: EM HPM100 and EM PACT2 (Leica Microsystems, Vienna, Austria), HPM 010 (Boeckeler Instruments, Tucson, AZ, USA), and HPF Compact 02 (Engineering Office M. Wohlwend, Sennwald, Switzerland). EM HPM100, HPM 010, and HPF Compact 02 are systems using liquid nitrogen at high pressure to pressurize and to cool the specimen in a small container located in the pressure chamber. A small volume of alcohol at room temperature is usually introduced first to coordinate pressure buildup and cooling. In the EM PACT2 system, the specimen in the container is pressurized with a transmission fluid (methylcyclohexane) and subsequently cooled with a stream of liquid nitrogen at 10 bars [1-3].

Several articles about HPF have been published in the last years. In particular, they focus on specimen preparation and handling before freezing, which are the most important steps to make HPF a successful method to preserve the ultrastructure of biological specimens [4-16]. Specimen processing after freezing is also extensively described in the literature for a variety of different specimens and applications. This chapter will focus on the recent developments regarding HPF machines, specimen carriers and methods rather than providing a review of previous articles. The recently developed HPF machine EM HPMI00 is described in detail. Handling and specimen preparation is discussed as far as different from the other systems (HPM 010 and HPF Compact 02). Additionally, an improved method for freezing cell culture monolayers grown on Sapphire discs with the EM PACT2 system is presented.

The EM HPMI00 is a largely automated system with an integrated workstation and binocular microscope (see Fig. la). The centerpiece is a versatile cartridge, consisting of highly durable polymer pieces (see Fig. 1b). The assembled cartridge holds the specimen carrier sandwich (or other container) and guides it automatically through the freezing process, finally releasing cartridge, carriers, and sample in liquid nitrogen. This guarantees that the whole freezing sequence from pickup of the specimen to liquid nitrogen is consistent and reproducible.

A wide range of different specimen geometries can be frozen with the according set of cartridge pieces and specimen carriers. In particular, samples of up to $5 \mathrm{~mm}$ in diameter can be processed, which facilitates handling before freezing considerably. The cartridge is made of a thermally insulating polymer leading to cooling of the sample and carriers rather than the surrounding cartridge and to optimized heat extraction from the sample. Additionally, the volume 

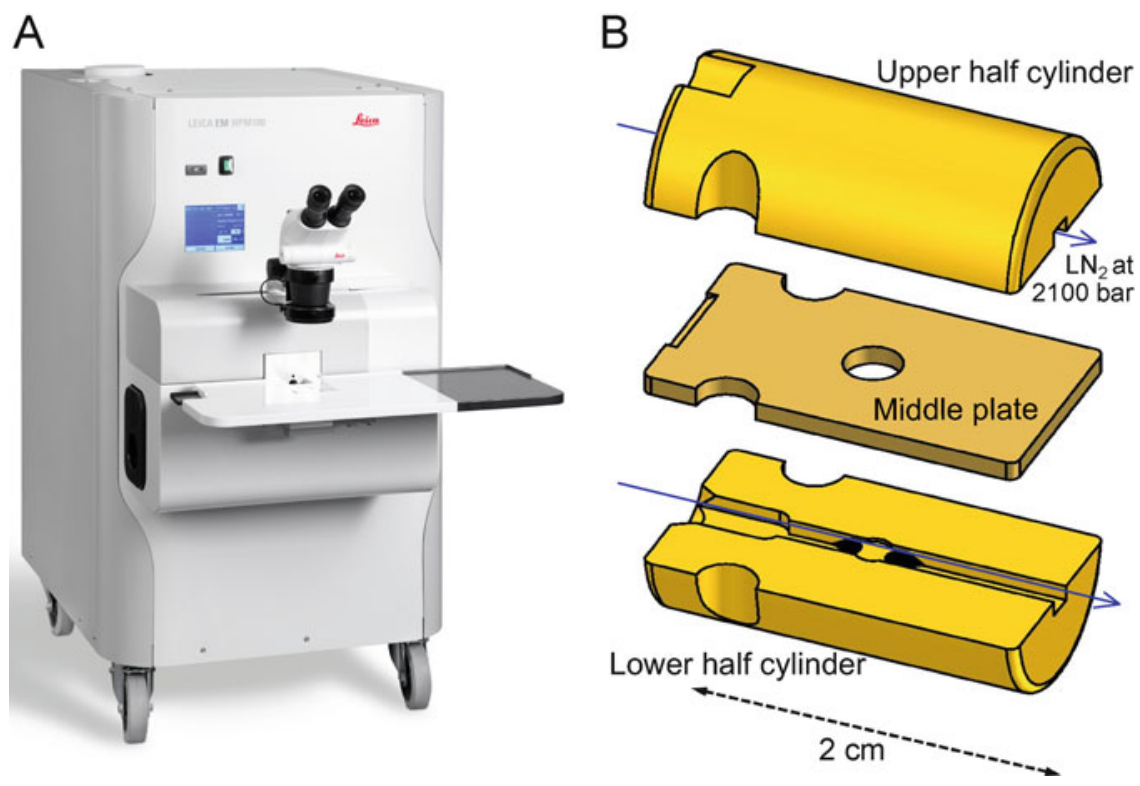

Fig. 1 (a) High-pressure freezing (HPF) machine EM HPM100. Front view showing workstation, binocular microscope and touch screen. (b) Exploded view of the cartridge system of the EM HPM100 for $3 \mathrm{~mm}$ specimen carriers: Identical upper and lower half cylinders and a middle plate with bore for the specimen carrier sandwich. Arrows indicate the flow of liquid nitrogen during the cooling process. Images courtesy of Leica Microsystems

of the pressure chamber was reduced compared to the previous HPM 010 model resulting in a quicker pressure buildup, higher flow rate of liquid nitrogen, and less nitrogen consumption.

Injection of alcohol at room temperature into the pressure chamber to coordinate pressure buildup and cooling can be switched off on demand. Application of this option will be discussed later in this chapter. A chamber to keep the temperature and humidity at desired values is available for the work station. The EM HPM100 is mobile and the remaining liquid nitrogen in the storage Dewar and pressure cylinder can be retrieved after a freezing session similar to the EM PACT2. Temperature and pressure courses are recorded and can be stored. The freezing quality and yield of well frozen samples are comparable to the results achieved with HPM $010[6,17-21]$.

The option of freezing specimens of a diameter up to $5 \mathrm{~mm}$ is appreciated by many scientists in the field and lead recently to adaptations also of HPM 010 and HPF Compact 02. Appropriate holders for up to $6 \mathrm{~mm}$ specimen carriers are now available for HPM 010 (per. comm. Bill Graham, Bibst Labs, Brookline, NH) and HPF Compact 02. An additional new feature of the HPF Compact 02 is that specimen holder containing the specimen carrier sandwich is now introduced horizontally into the pressure chamber compared to the vertical insertion with the predecessor model (per. comm. M. Wohlwend, Engineering Office M. Wohlwend, NH). 


\section{Materials}

\subsection{High-Pressure Freezing Machines}

\subsection{EM HPM100 Specimen Carriers and Cartridges}

2.2.1 $3 \mathrm{~mm}$ Aluminum Specimen Carrier System

2.2.2 6 mm Aluminum Specimen Carrier System

2.2.3 Cell Monolayers on $3 \mathrm{~mm}$ Sapphire Discs

2.2.4 Cell Monolayers on $6 \mathrm{~mm}$ Sapphire Discs
1. EM HPM100 with essential accessories (specimen carrier release device).

2. EM PACT2 with essential accessories (bayonet pod, loading station, rapid transfer system loading device, transfer slider, torque wrench).

Cartridges, specimen carriers and HPF machine associated parts are available from Leica Microsystems, else indicated. A wide variety of specimen carriers for all types of HPF machines are also available from Engineering Office M. Wohlwend.

1. $3 \mathrm{~mm}$ cartridge (half cylinders and middle plate).

2. $3 \mathrm{~mm}$ aluminum specimen carrier Type A $(100 \mu \mathrm{m} / 200 \mu \mathrm{m}$ recess).

3. $3 \mathrm{~mm}$ aluminum specimen carrier Type B (flat $/ 300 \mu \mathrm{m}$ recess).

4. $3 \mathrm{~mm}$ aluminum specimen carrier Type C (slit carrier, $100 \mu \mathrm{m} / 200 \mu \mathrm{m}$ recesses).

5. $3 \mathrm{~mm}$ aluminum specimen carrier with $150 / 150,275 / 25$, or $250 / 50 \mu \mathrm{m}$ recess (Engineering Office $M$. Wohlwend).

6. $3 \mathrm{~mm}$ punch for specimen carrier release device.

7. Round file to widen bore of the middle plate.

1. $6 \mathrm{~mm}$ cartridges (half cylinders and middle plate).

2. $6 \mathrm{~mm}$ aluminum specimen carrier Type A $(100 \mu \mathrm{m} / 200 \mu \mathrm{m}$ recess).

3. $6 \mathrm{~mm}$ aluminum specimen carrier Type B (flat $/ 300 \mu \mathrm{m}$ recess).

4. $6 \mathrm{~mm}$ aluminum specimen carrier with $150 / 150 \mu \mathrm{m}$ recesses (Engineering Office M. Wohlwend).

5. $6 \mathrm{~mm}$ punch for specimen carrier release device.

1. $3 \mathrm{~mm}$ Sapphire discs.

2. $6 \mathrm{~mm}$ cartridge half cylinders.

3 . $6 \mathrm{~mm}$ cartridge middle plate with edge.

4. $6 \mathrm{~mm}$ support rings for $3 \mathrm{~mm}$ Sapphire discs.

5. $3 \mathrm{~mm}$ aluminum specimen carrier Type B (flat $/ 300 \mu \mathrm{m}$ recess).

6. Cell culture dishes with optically appropriate glass or plastic bottom (MatTek Corporation, Ashland, MA, USA and ibidi GmbH, Planegg/Martinsried, Germany).

1. $6 \mathrm{~mm}$ Sapphire discs.

2. $6 \mathrm{~mm}$ cartridge half cylinders.

3. $6 \mathrm{~mm}$ cartridge middle plate with edge. 
2.2.5 Freeze-Fracturing of Thin Suspensions

\subsubsection{Cryo-sectioning Using Copper Specimen Tubes}

2.2.7 Cryo-sectioning Using Freeze-Fracture and Membrane Carrier

\subsection{EM PACT2 Carrier Systems}

2.3.1 Cell Monolayers on $1.4 \mathrm{~mm}$ Sapphire Discs

\subsection{Specimen} Preparation and Processing
4. $6 \mathrm{~mm}$ aluminum specimen carrier Type A $(100 \mu \mathrm{m} / 200 \mu \mathrm{m}$ recesses).

5. $6 \mathrm{~mm}$ spacer ring $200 \mu \mathrm{m}$ thickness.

6. $6 \mathrm{~mm}$ finder grid mask.

7. Cell culture dishes with optically appropriate glass or plastic bottom (MatTek Corporation and ibidi $\mathrm{GmbH}$ ).

1. $3 \mathrm{~mm}$ cartridges (half cylinders and middle plate).

2. $3 \mathrm{~mm}$ aluminum specimen carrier Type B (flat $/ 300 \mu \mathrm{m}$ recess).

3. Scalpel.

4. TEM grids of choice.

5. Round file to widen bore of the middle plate.

1. Cartridges for tubes (half cylinders).

2. Copper specimen tubes (outer diameter $0.65 \mathrm{~mm}$, inner diameter $0.35 \mathrm{~mm}$, length $9.5 \mathrm{~mm})$.

3. Tube press.

4. Tube holder for cryo-ultramicrotome.

1. $3 \mathrm{~mm}$ cartridges (half cylinders and middle plate).

2. Gold hat freeze-fracture carrier $3 \mathrm{~mm}$ with cylindrical recess, thickness $0.8 \mathrm{~mm}$.

3. Membrane carrier $2.8 \mathrm{~mm}$ diameter with recess of 100 or $200 \mu \mathrm{m}$.

4. Cryo-ultramicrotome holder for gold hat carrier (not yet available commercially) or cryo-glue and aluminum stubs.

1. Composite carrier consisting of gold ring and $1.4 \mathrm{~mm}$ Sapphire disc (available from Engineering Office M. Wohlwend).

1. Scalpel.

2. Razor blades.

3. Forceps, fine and blunt.

4. Tape.

5. Plastic Petri dishes.

6. 1.5 and $2 \mathrm{ml}$ Eppendorf tubes.

7. Diamond marking pen.

8. Millicell membrane inserts $(0.4 \mu \mathrm{m}$, Millipore, EMD Millipore Corporation, Billerica, MA, USA).

9. Vibratome. 
10. Biopsy punches ( 4 or $5 \mathrm{~mm}$ ).

11. Cellulose capillary tubes (Leica Microsystems).

12. 1-Hexadecene (e.g., 92 \%, Thermo Fisher Scientific, Waltham, MA, USA).

13. Yeast paste (Baker's yeast as thick suspension).

14. Dextran (MW 39,000, Sigma-Aldrich, St. Louis, MO, USA) solution $20 \%$ in an appropriate buffer for the sample.

15. Polylysine (Sigma-Aldrich).

\section{Methods}

\subsection{Loading of Samples and Handling Using the EM HPM100}

\subsubsection{Freezing Using the} 3 and $6 \mathrm{~mm}$ Aluminum Specimen Carrier System
For optimal results, the following basic rules for HPF are essential: Always use a fresh sample and as thin as possible, preferably thinner than $200 \mu \mathrm{m}$. Remove extracellular medium containing high amounts of water and substitute with a filler (e.g., l-hexadecene, yeast paste, Dextran solution). Make sure that no air is trapped in the specimen carriers.

Procedures for sample preparation as well as all specimen carrier sandwich configurations used with the HPF machines HPM 010 and HPF Compact 02 also apply to the EM HPM100. Cartridges are available for all specimen carriers used routinely with HPM 010 and HPF Compact 02 systems.

1. Place the three components of the cartridge for the 3 or $6 \mathrm{~mm}$ carrier system (upper, lower half cylinders and middle plate) in the correct position at the work station as shown in Fig. 2a, b. Upper and lower half cylinders are identical.

2. Prepare your specimen and assemble specimen carrier sandwich using the appropriate aluminum specimen carriers, fillers etc. [4-16].

3. Move the closed carrier sandwich containing the specimen using tweezers into the bore of the middle plate (see Fig. 2b). Alternatively, the sandwich can be assembled in the bore of the already mounted middle plate (see Note $\mathbf{1}$ ).

4. Flip the handle on the left side of the work station to complete the cartridge assembly containing carrier sandwich with specimen (see Fig. 2c, d).

Fig. 2 (continued) flipped to pick up the specimen and to complete the cartridge as depicted in (d). (e) Subsequently, the handle is flipped back and the process is started by pressing the safety button. After freezing, the cartridge with the frozen specimen is automatically transferred into the liquid nitrogen container in the drawer on the left side of the machine. (f) The specimen carrier sandwich is released from the middle plate by using the specimen carrier release device, which acts as a punch 

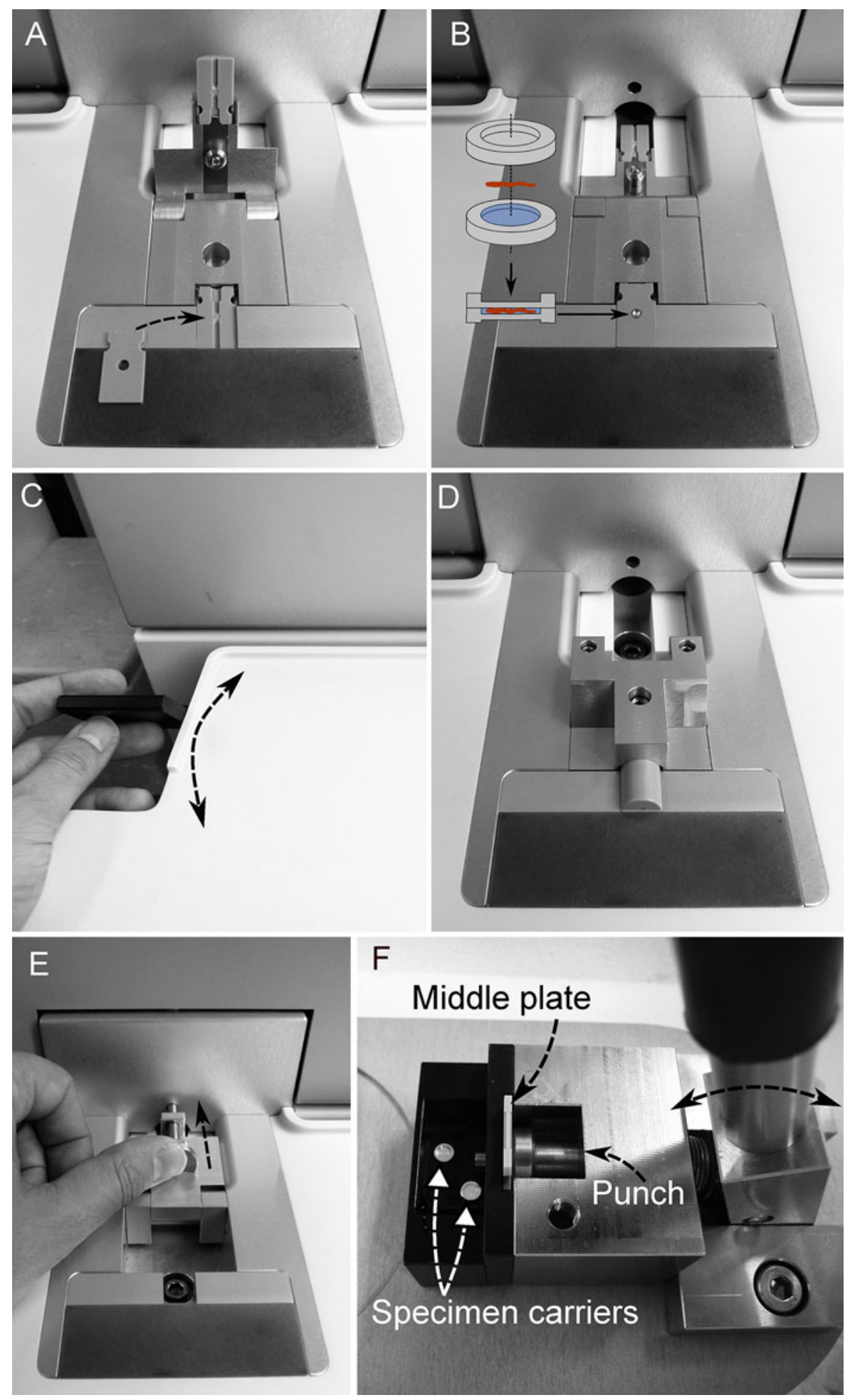

Fig. 2 Operations required for freezing samples with the EM HPM100. (a) Placing of the cartridge half cylinders at the work station. The arrow indicates the positioning of the middle plate. (b) $3 \mathrm{~mm}$ specimen carrier sandwich inserted into the recess of the positioned middle plate. (c) The handle on left side of the workstation is 
Tissue of Animals and Plants

Suspensions of Cells in Cellulose Capillary Tubes

High Viscosity Suspensions
5. Flip the handle backwards and press the safety lock at the front of the machine to start the freezing process (see Fig. 2e).

6. Remove the cartridge pieces and the specimen carriers from the liquid nitrogen Dewar in the drawer on the left side of the machine.

7. Punch out the specimen carrier sandwich from the middle plate using the specimen carrier release device if necessary (see Fig. $2 \mathrm{f}$ and Note 2 ).

The $6 \mathrm{~mm}$ aluminum specimen carrier system offers freezing of samples up to $5 \mathrm{~mm}$ in diameter. In most cases, only a small part of such a large sample will be needed for imaging in the electron microscope. However, the provided space for the specimen noticeably facilitates handling and loading of the sample before freezing in many cases (see following paragraphs).

The sizing of a sample before freezing is often demanding and may lead to damage of the specimen, which is of utmost importance to avoid. Additionally, the smaller the sample needs to be trimmed, the more difficult it gets to control the orientation. The interference of the operator with the sample or with the region of interest of the sample can be minimized using the $5 \mathrm{~mm}$ diameter space, e.g., when cutting slices with a scalpel or vibratome, punching leaf or tissue discs with up to $5 \mathrm{~mm}$ diameter biopsy punches ( see Note 3 ).

The large diameter is also beneficial for native tissue slice cultures cultivated on filter membranes, e.g., cerebellar slices of mice on Millicell membranes. The filter containing the whole tissue slice can be punched with a 4 or $5 \mathrm{~mm}$ biopsy punch and transferred using tweezers to the appropriate recess of a $6 \mathrm{~mm}$ aluminum specimen carrier (see Note 4 ).

This approach is also suitable for light microscopy imaging prior to HPF. The area of interest can be found easily also after freezesubstitution and embedding, since the complete slice with its original structure is still visible and accessible. The micrograph in Fig. 3 shows an area of a cerebellar slice processed using this method.

Cellulose capillary tubes are mainly used to freeze cell suspensions [22]. The capillaries containing the suspensions are immersed in a buffer solution or 1-hexadecene and are cut to a length of about $4 \mathrm{~mm}$ using a razor blade or scalpel. Due to their length, cutting can be carried out without the aid of a binocular microscope and the ends do not have to be closed. Capillaries are easily transferred with forceps to the recess (preferably $150 \mu \mathrm{m}$ ) of a $6 \mathrm{~mm}$ aluminum specimen carrier.

Depending on the nature of cells or their treatment, suspensions (e.g., bacteria) often form aggregates which cannot be pipetted or drawn into capillaries. They need to be transferred to the recess of the specimen carrier with a spatula, for example. This transfer is facilitated remarkably when using $6 \mathrm{~mm}$ specimen carriers. 


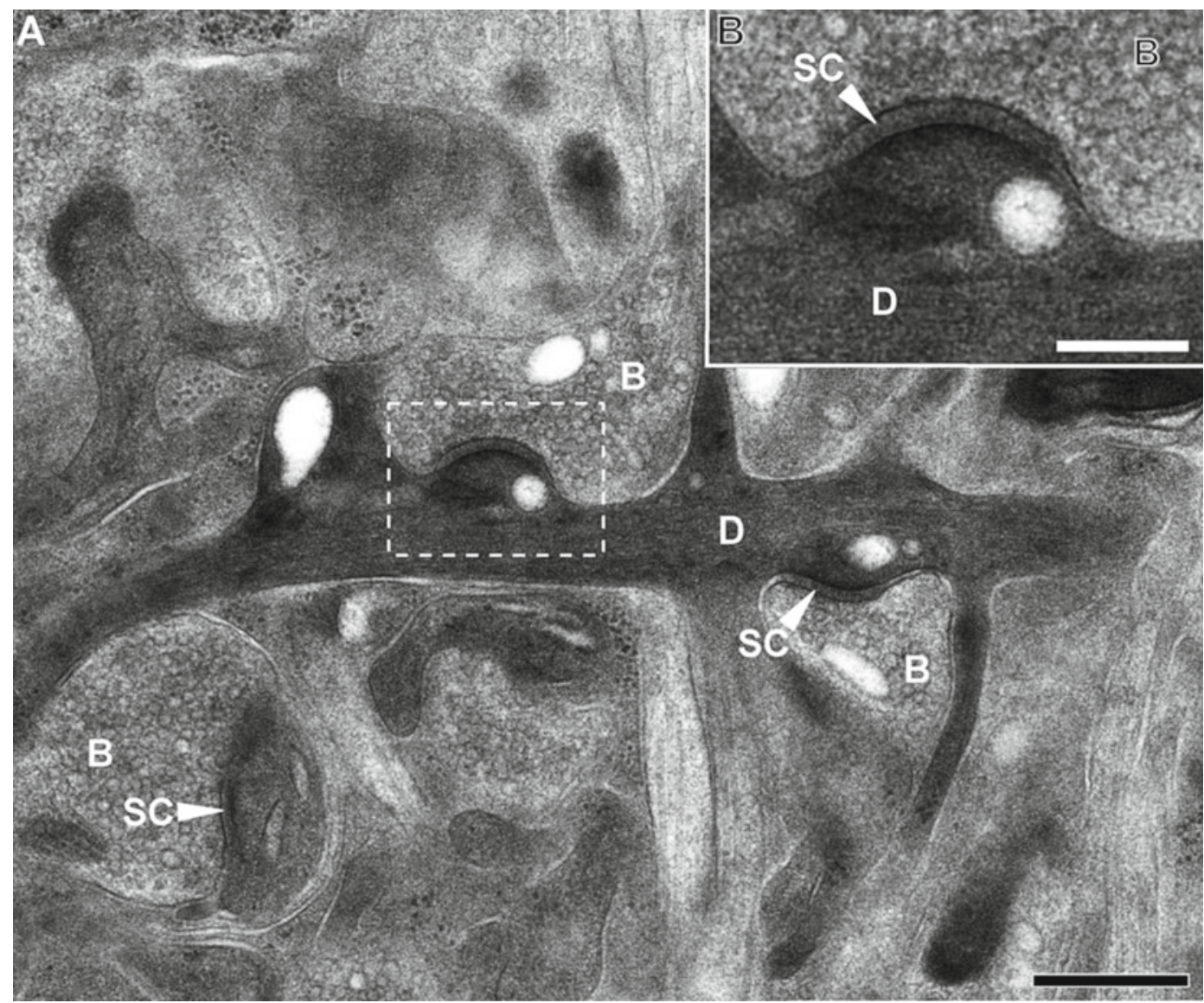

Fig. 3 Synaptic boutons at a dendrite of a cerebellar slice culture of mouse, high-pressure frozen in $6 \mathrm{~mm}$ aluminum specimen carriers (100 $\mu \mathrm{m}$ recess). The specimen was freeze-substituted in acetone containing $2 \% \mathrm{OsO}_{4}$ and embedded in Epon/Araldite. (b) Higher magnification of the marked area in (a). B Synaptic bouton with synaptic vesicles, $D$ Dendrite, SC Synaptic cleft. Scale bars: $(\mathbf{a})=500 \mathrm{~nm} ;(\mathbf{b})=200 \mathrm{~nm}$

Implications of $6 \mathrm{~mm}$

Carriers on

Freeze-Substitution

\subsubsection{Freezing Cell}

Culture Monolayers on 3 and $6 \mathrm{~mm}$ Sapphire Discs
$6 \mathrm{~mm}$ aluminum specimen carriers require appropriate tubes for freeze-substitution to ensure sufficient diffusion of the substitution solution. For example, they can divide the volume of a cone shaped $1.5 \mathrm{~mm}$ Eppendorf tube into two separate reservoirs inhibiting efficient substitution. It is recommended to use cylindrical $2 \mathrm{ml}$ tubes or other appropriate containers. Also use enough volume of freeze-substitution medium. The volume of the recess $(200 \mu \mathrm{m}$ depth) of a $6 \mathrm{~mm}$ aluminum carrier is more than six times larger than the one of a $3 \mathrm{~mm}$ aluminum specimen carrier with same recess.

HPF of cell culture monolayers grown on Sapphire discs is a widely used method for the investigation of their ultrastructure [23-25]. Sapphire discs often are coated with a layer of carbon $(5-20 \mathrm{~nm})$ or polylysine providing a good substrate for cell proliferation. In particular, a carbon layer with an asymmetric mark facilitates handling considerably due to better visibility and unambiguous identification 
of the cell side ( see Note 5). Alternatively, a mark can also be added to the bare Sapphire disc using a diamond marking pen.

Different sandwich combinations using aluminum specimen carriers and spacer rings are possible to freeze cell monolayers on Sapphire discs and were described for the HPM 010 and EM Compact 02 type of high-pressure freezers [9]. All combinations can be still employed with the EM HPM100 high-pressure freezer. However, it has to be taken into account that the Sapphire discs have a very low mass compared to the aluminum specimen carriers and that they are surrounded by the warm middle plate and half cylinders during the automated transfer to the liquid nitrogen after the freezing process. In particular, the approach of using only spacers and two Sapphire discs $(0.5 \mathrm{~mm}$ spacer, Sapphire disc, $50 \mu \mathrm{m}$ spacer, Sapphire disc, $0.5 \mathrm{~mm}$ spacer) bears the risk of thawing the cells during the transfer. Therefore, the following simple setups for freezing cell cultures grown on 3 and $6 \mathrm{~mm}$ Sapphire discs were developed (see Fig. 4). The $6 \mathrm{~mm}$ Sapphire discs facilitate handling before freezing including imaging by light microscopy and during all subsequent preparation steps, e.g., freeze-substitution and embedding. Due to the large diameter, the Sapphire discs can be manipulated safely without damaging the cells. An example is given in Fig. 4e.

1. Switch off alcohol injection in the system page of the EM HPM100 software and make a few test shots to remove all excess alcohol left in the system (see Note 6).

2. Place the two half cylinders of the $6 \mathrm{~mm}$ system in the correct position at the work station.

3. Prepare a loading station for the middle plate with edge, e.g., stick two strips of a few layers of regular tape onto a plastic Petri dish and put the middle plate like a bridge across the two strips (see Fig. 4a, b). The middle plate should be kept slightly elevated from the preparation table to prevent capillary forces drawing off the cell culture liquid and drying of the cells.

4. a. When freezing $6 \mathrm{~mm}$ Sapphire discs: Insert the $6 \mathrm{~mm}$ Sapphire disc with the cells facing up onto the edge of the middle plate. Cover with a $6 \mathrm{~mm}$ aluminum specimen carrier Type A with the $100 \mu \mathrm{m}$ recess facing the cells (wetted with 1-hexedecene or other filler). Add a $200 \mu \mathrm{m}$ spacer ring

Fig. 4 (continued) thickness is added on top to fill up the recess of the middle plate. (d) Specimen carrier assembly for freezing cell monolayers on $3 \mathrm{~mm}$ Sapphire discs. A support ring is inserted into the recess of a $6 \mathrm{~mm}$ middle plate with edge. The $3 \mathrm{~mm}$ Sapphire disc is placed in the recess of the support ring with the cells facing up and covered with a flat $6 \mathrm{~mm}$ aluminum specimen carrier with the flat side facing down. (e) HEP-2 cell infected with an obligate intracellular bacterium Chlamydia pneumoniae. Magnification of the area marked in (f). Cells were cultured on $6 \mathrm{~mm}$ Sapphire discs. The specimen was freeze-substituted in acetone containing $2 \% \mathrm{OSO}_{4}$ and embedded in Epon/Araldite. C Chlamydia (darker forms are elementary bodies), ER endoplasmatic reticulum, $I n$ inclusion body, $M$ mitochondria, $N$ nucleus. Scale bars: $(\mathbf{e})=1 \mu \mathrm{m} ;(\mathbf{f})=5 \mu \mathrm{m}$ 
A

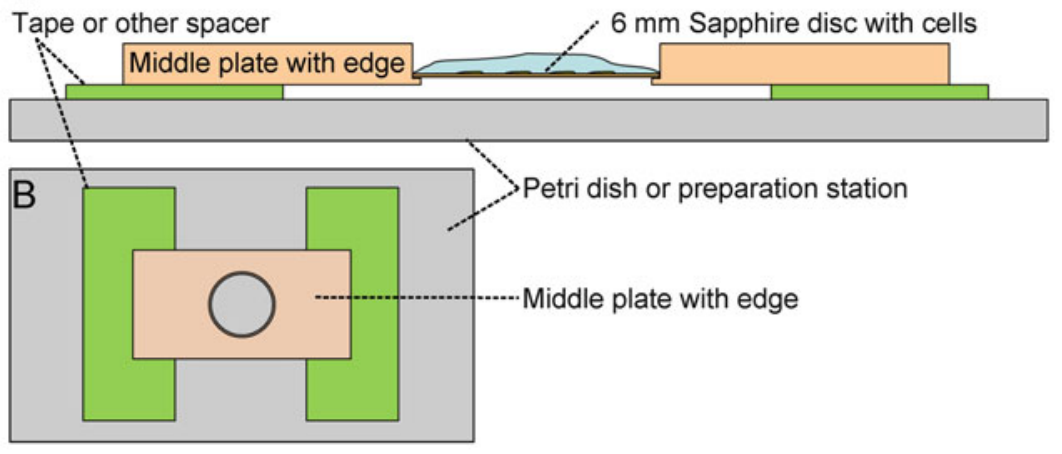

C

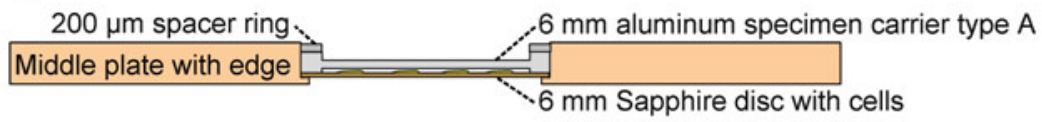

D
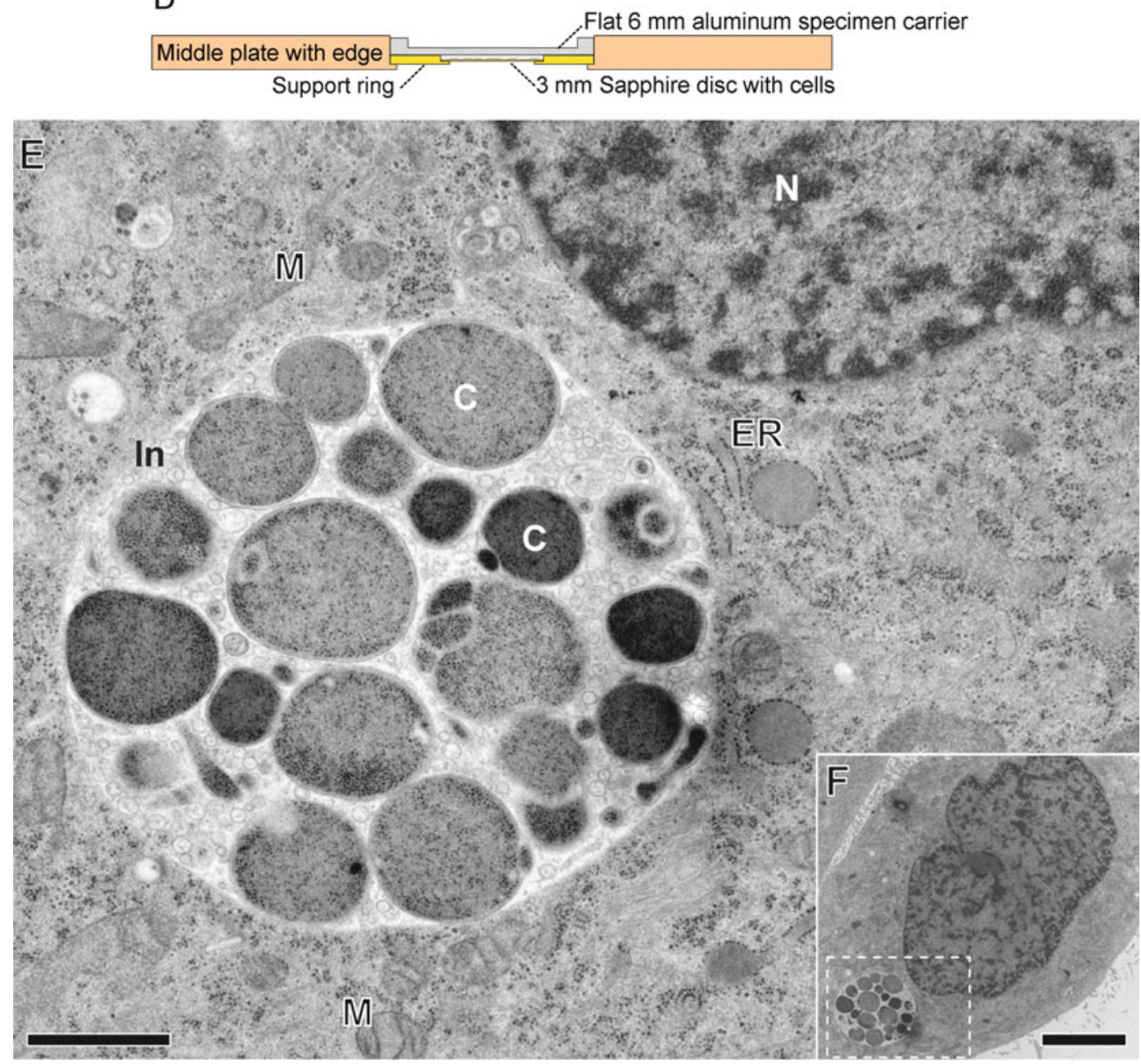

Fig. 4 (a) Setup for loading Sapphire discs containing cell monolayers into $6 \mathrm{~mm}$ middle plates with edge (shown for $6 \mathrm{~mm}$ Sapphire disc), side view. Elevation of the middle plate prevents culture liquid to be drawn off by capillary forces. (b) Reduced top view. (c) Specimen carrier assembly for freezing cell monolayers on $6 \mathrm{~mm}$ Sapphire discs. The Sapphire disc is inserted into the recess of a six middle plate with edge with the cells facing up and covered with the $100 \mu \mathrm{m}$ cavity of a $6 \mathrm{~mm}$ aluminum specimen carrier Type A. A $6 \mathrm{~mm}$ spacer ring of $200 \mu \mathrm{m}$ 
Considerations Regarding Light Microscopy Imaging Prior to Freezing

Freeze-Substitution and Embedding of Cell Monolayers on $6 \mathrm{~mm}$ Sapphire Discs

\subsubsection{Freezing of Suspensions for Subsequent Freeze- Fracturing Using $3 \mathrm{~mm}$ Aluminum Specimen Carriers and TEM Grids as Spacers}

on top of the aluminum specimen carrier to fill up the recess of the middle plate (see Fig. $4 \mathrm{c}$ and Note 7).

b. When freezing $3 \mathrm{~mm}$ Sapphire discs: Add the support ring onto the edge of the middle plate. Insert the $3 \mathrm{~mm}$ Sapphire disc with the cells facing up into the recess of the support ring. Cover with a flat $6 \mathrm{~mm}$ aluminum specimen carrier (wetted with 1 -hexadecene) with the flat side facing the Sapphire disc (see Fig. 4d).

5. Freeze the sample.

6. Use blunt tweezers to remove spacer, aluminum carrier, support ring, and Sapphire disc from the middle plate. Push carefully from the back side.

High-resolution fluorescence imaging of adherent cells prior to freezing can be obtained by putting the Sapphire disc with cells facing down in a dish (preferably separated from dish bottom with a spacer of $\sim 50 \mu \mathrm{m}$ ) containing cell culture medium and equipped with optically appropriate glass or plastic bottom. This simple setup allows imaging on an inverted microscope using high-resolution immersion objectives even during long term experiments (incubation chamber and $\mathrm{CO}_{2}$ supply required). For HPF, the Sapphire disc needs to be transferred into the cartridge middle plate manually with tweezers and the carrier assembly has to be completed according to the procedures for 3 and $6 \mathrm{~mm}$ Sapphire discs described above. This procedure requires approximately $30 \mathrm{~s}$ and, hence, does not allow capture of short-term processes. To our knowledge, no routine method for fast and ultrafast processes $(<10 \mathrm{~s})$ is developed to date.

Freeze-substitution of $6 \mathrm{~mm}$ Sapphire discs can be performed in cylindrical $2 \mathrm{ml}$ tubes. However, for the final embedding step, the Sapphire discs are preferably transferred to a cone shaped $1.5 \mathrm{ml}$ tube and aligned horizontally with the cells facing up. After polymerization, the tube is cut away and the resin block can be easily split in two pieces, the upper part containing the cell layer which can be trimmed right away ( see Note 8 ).

Freeze-fracturing of high-pressure frozen samples is usually performed by sandwich fracturing [26]. A random fracture face is produced by hitting off one carrier of the complete specimen carrier sandwich rather than creating fracture faces by using the knife in the freeze-fracturing machine (see Note 9).

The challenge of freezing samples for subsequent freeze-fracturing in most cases is to keep the carrier sandwich together after freezing and preventing pre-fracturing. An adequate sandwich configuration for the chosen specimen is required and depends on the desired application, e.g., imaging replicas by TEM 


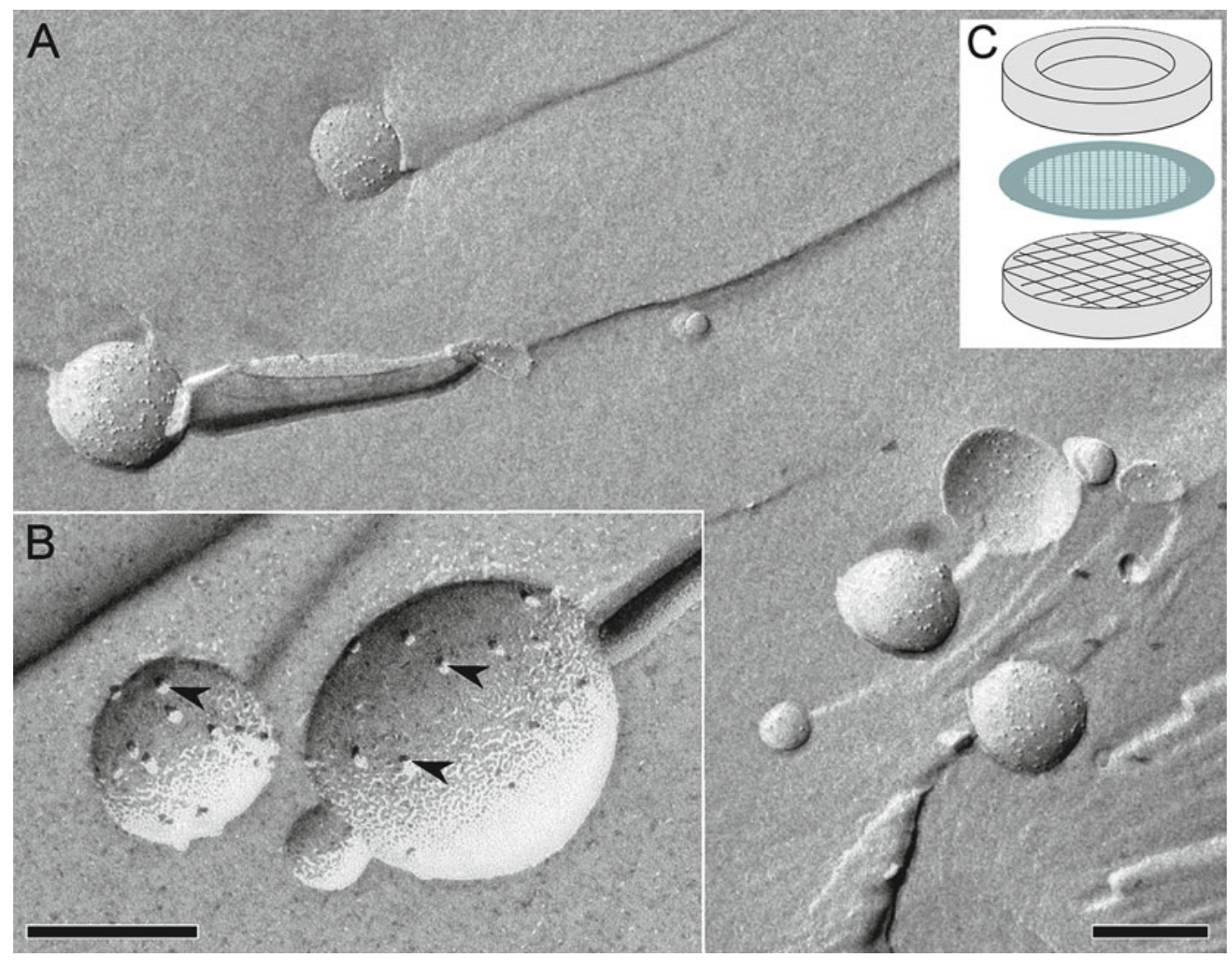

Fig. 5 (a and $\mathbf{b}$ ) Replica of high-pressure frozen and freeze-fractured liposome suspension showing reconstituted chloride proton antiporter proteins from Escherichia coli on fractured surfaces (arrow heads). (c) Exploded view of the specimen carrier assembly for freezing suspensions for subsequent freeze-fracturing. The TEM grid containing the suspension is sandwiched between two scored flat aluminum specimen carriers. Scale bars: (a) and (b) $=100 \mathrm{~nm}$. Specimen courtesy of Juan-Jose Garcia-Celma, Institute of Biochemistry, University of Zurich, Switzerland

or imaging by cryo-SEM. For a variety of specimens like animal or plant tissues, modified or even specially designed specimen carriers may be necessary to achieve successful processing. Several methods to freeze samples using HPF for subsequent freeze-fracturing were described previously [9] and can also be applied using the EM HPMl00 including the $4.6[7,27]$ and $6 \mathrm{~mm}$ specimen carrier systems. Here, only one of several methods for fracturing suspensions is presented (see Fig. 5), because it requires a modification of the cartridge middle plate.

1. Switch off alcohol injection in the system page of the EM HPM100 software and make a few test shots to remove all excess alcohol left in the system ( see Note 10).

2 . Widen the bore of the $3 \mathrm{~mm}$ middle plate to $3.1-3.2 \mathrm{~mm}$ using a round file. This is essential since the diameter of the grids $(3.05 \mathrm{~mm})$ is slightly larger than the bore of the middle plate. 
3.1.4 Freezing Samples for Cryo-sectioning Using the Tube System
3.1.5 Freezing Samples for Cryo-sectioning Using the Sandwich Method
3. Score the flat surface of the $3 \mathrm{~mm}$ aluminum specimen carriers (Type B) with a scalpel (see Note 11).

4. Place the three components of the cartridge (upper, lower half cylinders and middle plate) in the correct position at the work station.

5. Add a flat $3 \mathrm{~mm}$ aluminum specimen carrier into the bore of the middle plate with the flat, scored surface facing upwards.

6. Dip a TEM grid (material, mesh size and thickness of choice) into a concentrated suspension and add on top of the flat aluminum carrier ( see Note 12).

7. Cover with a second flat $3 \mathrm{~mm}$ aluminum specimen carrier with scored surface facing the grid (see Fig. 5c).

8. Freeze the specimen.

9. Remove the cartridge pieces and the specimen sandwich from the liquid nitrogen Dewar (see Note 13).

The copper tubes used for freezing with the EM HPM100 have the same inner $(350 \mu \mathrm{m})$ and outer $(650 \mu \mathrm{m})$ diameter as the tubes used in the EM PACT2 but are of different length $(9.5 \mathrm{~mm})$. The handling for mounting in the cryo-ultramicrotome and sectioning is identical. The cartridge for the tubes consists of two equal half cylinders (no middle plate) which hold the copper tube containing the specimen in place during the freezing process. Alcohol injection can be optionally switched on for this application. This system provides ambiguous results regarding the reliability of freezing quality (per. comm. Markus Grabenbauer, MPI, Dortmund, Germany, 2012).

1. Place appropriate upper and lower half cylinders in the correct position at the work station.

2. Fill the copper tube with the specimen suspension using a pipette with a $200 \mu \mathrm{l}$ tip. The copper tube can be easily attached at the pipette tip because the opening diameter of the $200 \mu \mathrm{l}$ tip is slightly smaller than the diameter of the copper tube.

3 . Close the copper tube at one side using the specially designed tube press or flat nose pliers.

4. Transfer the tube to the recess of the half cylinder with the closed end facing towards you.

5 . Freeze the specimen.

6. Remove the tube from the liquid nitrogen Dewar.

The following approach was developed by Kim Rensing (Leica Microsystems) and is a promising method for cryo-sectioning of suspensions and tissues.

1. Switch off alcohol injection in the system page of the EM HPM100 software and make a few test shots to remove all excess alcohol left in the system. 


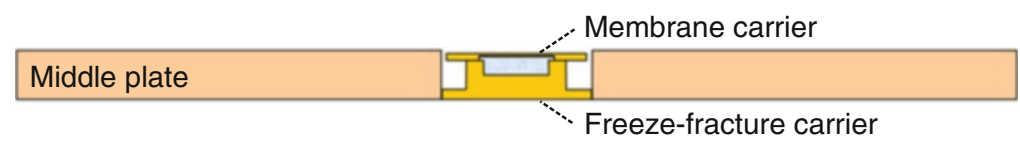

Fig. 6 Specimen carrier assembly for freezing samples for subsequent cryosectioning. The specimen is sandwiched between a freeze-fracturing carrier with cylindrical recess and a membrane carrier. After freezing, the membrane carrier is removed and the freeze-fracturing carrier containing dome-shaped specimen is mounted onto the arm of the cryo-ultramicrotome for cryo-sectioning

2. Place the three components of the cartridge for the $3 \mathrm{~mm}$ aluminum specimen carrier system in the correct position at the work station.

3 . Put a $3 \mathrm{~mm}$ freeze-fracturing specimen carrier with cylindrical recess (total thickness $0.8 \mathrm{~mm}$ ) into the bore of the $3 \mathrm{~mm}$ middle plate.

4. Transfer the sample (suspension or tissue piece) into the cylindrical recess of the carrier. Make sure that the sample is sticking out of the recess by 100-200 $\mu \mathrm{m}$.

5 . Wet a 100 or $200 \mu \mathrm{m}$ membrane carrier with 1 -hexadecene.

6. Complete the sandwich by adding the membrane carrier on top with the recess facing the specimen (see Fig. 6).

7. Freeze the specimen.

8. Remove the sandwich from the liquid nitrogen Dewar.

9. Remove the membrane carrier carefully so that the specimen sticks out of the bottom carrier (see Note 14).

The $3 \mathrm{~mm}$ bottom carrier can be attached in the cryoultramicrotome either on a standard aluminum stub using cryo-glue [28] or mechanically with an adapted $3 \mathrm{~mm}$ clamp system (not yet commercially available). Cryo-sectioning is performed close to the surface previously covered by the membrane carrier during freezing and, hence, where vitrification is primarily achieved.

3.2 An Advanced Technique to Freeze Cell Culture Monolayers on Sapphire Discs Using the EM PACT2 High-Pressure Freezer
Sapphire discs used for freezing monolayers of cell cultures with the EM PACT2 have a diameter of $1.4 \mathrm{~mm}$ only. Due to the very small diameter of the discs the handling during preparation, transfer for freezing, freeze-substitution and embedding is cumbersome. We developed a technique which facilitates handling considerably and consistently provides enhanced freezing quality. A specific composite carrier is required for this application, consisting of a gold ring and a $1.4 \mathrm{~mm}$ Sapphire disc (see Fig. 7a).

Composite carriers can be subjected to all standard cell culture procedures like cleaning with ethanol, coating with carbon and sterilizing by heat or UV irradiation, but are also easy to handle without touching or damaging the cells with tweezers. A further advantage is that the orientation of the assembly can be discerned by eye easily. 
A

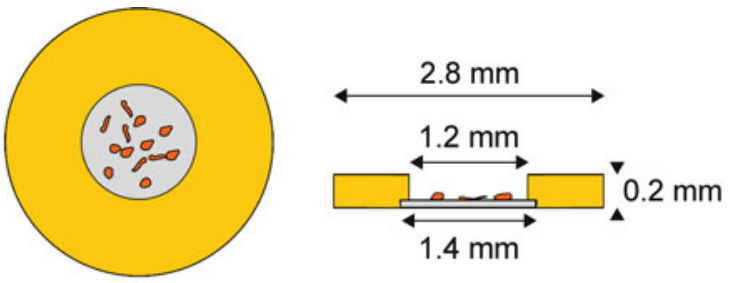

B
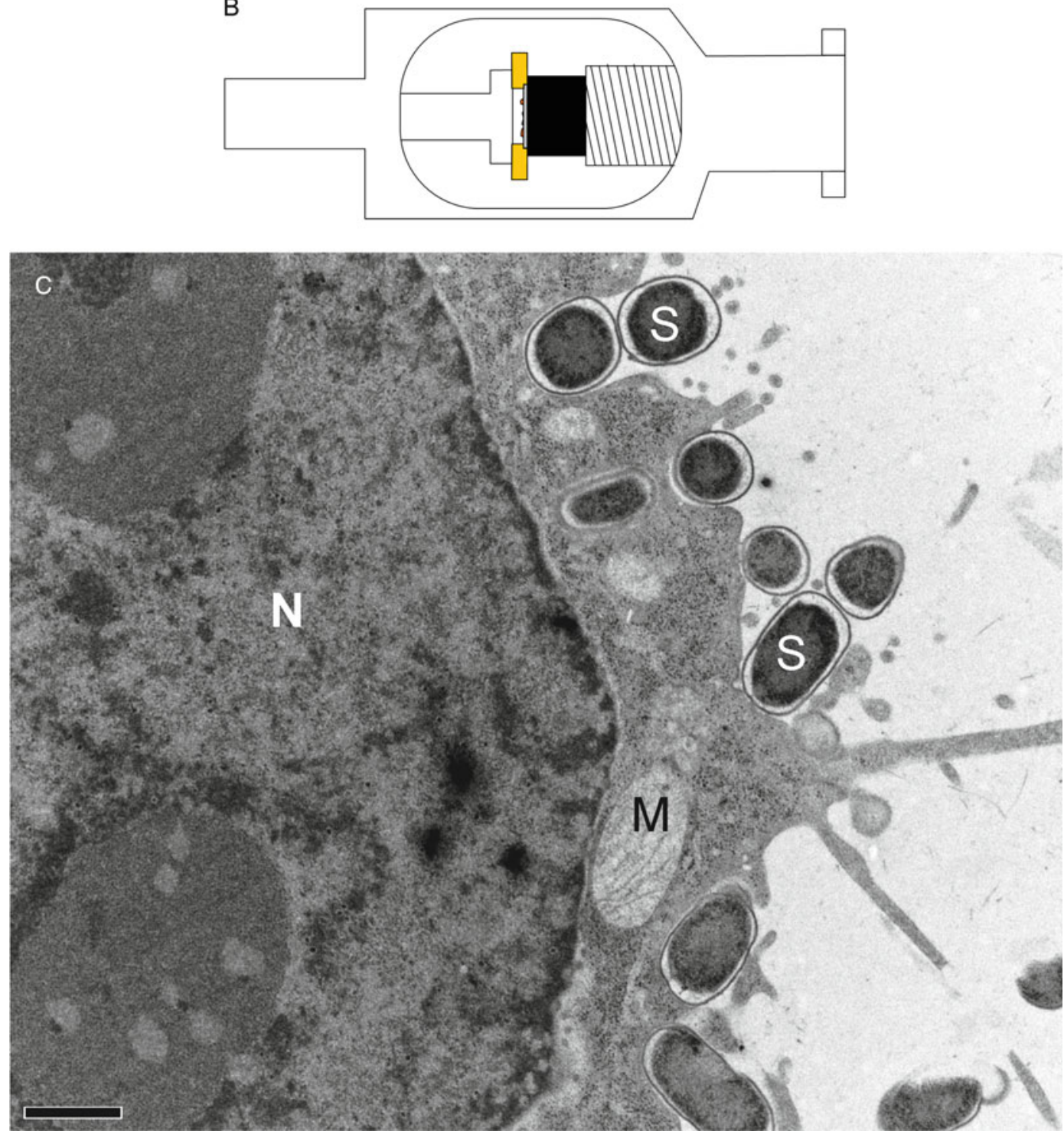

Fig. 7 (a) Top and side view of the composite carrier made of a gold ring and a Sapphire disc. (b) Schematic drawing of the composite carrier attached in the freezing pod. The diamond of the freezing pod contacts the Sapphire disc directly and permits optimum heat transfer. (c) Hela cell infected with an enterobacterium Salmonella typhimurium, high-pressure frozen using composite carriers. The specimen was freeze-substituted in acetone containing $2 \% \mathrm{OsO}_{4}$ and embedded in Epon/Araldite. $N$ nucleus, $S$ Salmonella, $M$ mitochondrium. Scale bar $=1 \mu \mathrm{m}$. Specimen courtesy of Sabrina Dilling, Institute of Microbiology, ETH Zurich, Switzerland 
1. Coat the composite carrier with material of choice, e.g., carbon layer and gold finder replica. Make sure that the recess of the carrier is facing up.

2. Sterilize composite carrier by UV light or dry autoclaving.

3. Add the sterilized composite carrier to the cell culture dish and incubate cells according to the desired conditions ( see Note 15).

4. Remove the composite carrier from the cell culture dish and transfer upside down onto a droplet of medium on a flat surface. Pick up the carrier quickly with the slider. Alternatively, the composite carrier can also be flipped in the cell culture dish and transferred directly into the spring of the slider using forceps.

5. Slide into the bayonet pod on the loading station, close with torque wrench, pick up the pod (see Fig. 7b) with the manual bayonet loading device and freeze the sample. When using the rapid transfer system (RTS), pick up the sample with the RTS slider and freeze the specimen (see Notes 16 and 17).

6. Proceed with freeze-substitution and embedding. Embed whole composite carrier. The gold ring can be trimmed away after polymerization and the Sapphire disc split off with the help of liquid nitrogen if necessary.

An example is given in Fig. $7 c$.

\section{Notes}

1. If the sample carrier is assembled directly in the middle plate, make sure to keep the bottom carrier filled properly with liquid and/or specimen before completing the sandwich and proceed quickly for freezing. Capillary forces between carrier and the middle plate can draw off extracellular liquids or suspensions leading to a non-completely filled carrier sandwich and hence to unsuccessful freezing and bent carriers. You may also use the following workaround: The $3 \mathrm{~mm}$ recess in the middle plate can be widened to approximately 3.1 or $3.2 \mathrm{~mm}$, e.g., with a cylindrical file. The additional gap between carrier and middle plate helps to prevent the capillary forces drawing off liquids surrounding the specimen.

2. When using alcohol for coordination of pressure buildup and cooling the specimen sandwich is kept very tightly in the bore of the middle plate due to frozen alcohol.

3. Although biopsy guns are available (EM PACT2 biopsy system, biopsy gun and needles for EM HPM100 or any high-pressure freezer), their use is still unsatisfying. Usually, the required region of interest of an organ cannot be excised precisely, transfer to the carrier is tedious, and the sausage-like geometry of the biopsy makes orienting of the sample almost impossible 
during the processing steps for electron microscopy. In addition, the dimensions of the indentation of the needles are too wide, large and long to lead to a high yield of non-distorted, well-frozen samples. A good compromise is perfusion of the animal/organ with fixatives (e.g., 3 \% formaldehyde and $0.5 \%$ glutaraldehyde) and dissection prior to HPF. Excision of the region of interest is usually carried out in $2 \%$ glutaraldehyde solution. Optionally, the tissue pieces can be immersed in a solution containing antifreeze (e.g., $20 \%$ glycerol) before freezing $[5,29,30]$.

4. Alternatively, pre-punched filter discs can be used to cultivate the tissue slices (e.g., brain) on the Millicell membrane. Filter discs can be transferred directly to the specimen carrier (no punching required).

5. Sterilization of the carbon coated Sapphire disc is best done by dry heat sterilization, since the process stabilizes the carbon layer. A finder grid replica can be applied on top of the carbon by sputter coating with $15 \mathrm{~nm}$ of gold. A replica is essential to analyze a certain cell by electron microscopy which was selected or imaged primarily by light microscopy.

6. Excess alcohol freezes at the edge of the middle plate or support ring and makes it impossible to remove the Sapphire disc from the middle plate after freezing without damage. Punches of the sample carrier release device cannot be used because of the edge of the $6 \mathrm{~mm}$ middle plate. If you use alcohol during the freezing process for some reason, keep the Sapphire disc in the middle plate until processing by freeze-substitution. When immersing the middle plate in acetone at $-90^{\circ} \mathrm{C}$, the Sapphire discs can easily be removed from the middle plate after a few seconds and transferred into the freeze-substitution solution. This works best when using ethanol due to its melting temperature of $-114^{\circ} \mathrm{C}$, compared to the melting point of isopropanol at $-89.5^{\circ} \mathrm{C}$. In our experience, the freezing quality varies in the same range when using ethanol as synchronization fluid or omitting it.

7. Omitting the top spacer often leads to broken Sapphire discs.

8. A special basket system for freeze-substitution of cell cultures on $6 \mathrm{~mm}$ Sapphire discs is available (Leica Microsystems). It is also applicable for freeze-substitution of samples frozen in $6 \mathrm{~mm}$ aluminum specimen carriers.

9. High-pressure frozen samples are very brittle making it difficult to fracture with a knife without removing the sample from the carrier or breaking it into many tiny pieces by accident. Moreover, it is delicate to prepare a sample sandwich for freezing which allows removing the cover carrier after freezing and at 
the same time keeping the sample tightly and completely in the bottom carrier.

10. In general, it is suggested to omit alcohol injection in the EM HPM100 when freezing samples for freeze-fracturing. Alcohol will freeze at the sandwich surfaces and keep it tightly in the middle plate of the cartridge. Punching out the carrier sandwich in the sample carrier release device leads to pre-fracturing in most cases.

11. The scored surface helps to keep the frozen sandwich together and to increase the chance of getting a fracture face through the sample rather than along the surface of the aluminum specimen carriers. Fractured material can be always found in the grooves of the aluminum carriers, e.g., when fracturing small particles like liposomes.

12. With regular TEM grids of approximately $12 \mu \mathrm{m}$ thickness, the method works for thin suspensions like cell cultures, bacteria, or liposomes. For larger specimen dimensions and structures, choose appropriate grid thickness or apply another sandwich configuration [9].

13. The specimen sandwich usually is found separately in the liquid nitrogen Dewar. The yield of sandwiches kept together after freezing depends strongly on the properties of the specimen.

14. The best chance to obtain a dome sticking out of the bottom carrier after freezing is achieved with dense suspensions, usually centrifuged in a $20 \%$ Dextran (MW 39,000) solution in buffer.

15. Place the composite carrier in the cell culture well before adding the cell suspension. Based on our experience, we recommend putting a small droplet of the cell suspension on top of the composite carrier first in order to achieve a high number of cells attaching to the Sapphire disc.

16. Fill the bore of the bayonet pod always (manual handling or RTS) with 1-hexadecene to make sure that no air is left in the system during freezing. Also make sure that enough cell culture liquid is left on top of the cells after picking up the carrier with the slider. Optionally, dip tip of slider into 1-hexdecene to prevent drying of the sample and keeping recess of the composite carrier completely filled.

17. The diamond cylinder of the freezing pod contacts the whole surface of the Sapphire disc and allows optimum heat transfer. Superb freezing is achieved, because the heat transfer between cells and liquid nitrogen is mainly mediated through the Sapphire rather than through filler and carrier. 


\section{Acknowledgements}

We would like to thank all colleagues in the field for fruitful and stimulating discussions and input, which were and still are indispensable for improving the application and technique of HPF. Special thanks go to Kim Rensing for sharing his experience and developed methods and for proofreading the manuscript. We wish also to thank all our collaborators for providing so many different and challenging samples.

\section{References}

1. Studer D, Graber W, Al-Amoudi A et al (2001) A new approach for cryofixation by high-pressure freezing. J Microsc (Oxford) 203:285-294

2. Riehle U (1968) Über die Vitrifizierung verdünnter wässriger Lösungen. Thesis, Federal Institute of Technology, Zurich, Switzerland.

3. Moor H, Riehle U (1968) Snap-freezing under high pressure: a new fixation technique for freezeetching. In: Bocciarelli DS (ed) Proceedings of the fourth European regional conference on electron microscopy vol 2. Rome, pp 33-34

4. McDonald K, Schwarz H, Muller-Reichert T et al (2010) "Tips and tricks" for high-pressure freezing of model systems. Methods Cell Biol 96:671-693

5. Muhlfeld C (2010) High-pressure freezing, chemical fixation and freeze-substitution for immuno-electron microscopy. Methods Mol Biol 611:87-101

6. Kang BH (2010) Electron microscopy and high-pressure freezing of Arabidopsis. Methods Cell Biol 96:259-283

7. Möbius W, Cooper B, Kaufmann WA et al (2010) Electron microscopy of the mouse central nervous system. Methods Cell Biol 96:475-512

8. Vanhecke D, Graber W, Studer D (2010) Rapidly excised and cryofixed rat tissue. Methods Cell Biol 96:513-527

9. Kaech A (2008) BAL-TEC HPM 010 highpressure freezing machine. In: Cavalier A, Spehner D, Humbel BM (eds) Handbook of Cryo-Preparation Methodsfor Electron Microscopy. CRC Press, Boca Raton, FL, pp 101-128

10. Studer D, Vanhecke D (2008) High-pressure freezing Leica Empact. In: Cavalier A, Spehner D, Humbel BM (eds) Handbook of CryoPreparation Methods for Electron Microscopy. CRC Press, Boca Raton, FL, pp 131-156

11. Vanhecke D, Graber W, Studer D (2008) Close-to-native ultrastructural preservation by high pressure freezing. Methods Cell Biol 88:151-164
12. Murray S (2008) High pressure freezing and freeze substitution of Schizosaccharomyces pombe and Saccharomyces cerevisiae for TEM. Methods Cell Biol 88:3-17

13. Reipert S, Wiche G (2008) High-pressure freezing and low-temperature fixation of cell monolayers grown on sapphire coverslips. Methods Cell Biol 88:165-180

14. McDonald K (2007) Cryopreparation methods for electron microscopy of selected model systems. Methods Cell Biol 77:23-56

15. Hess MW (2007) Cryopreparation methodology for plant cell biology. Methods Cell Biol 77:57-100

16. McDonald KL, Morphew M, Verkade P et al (2007) Recent advances in high-pressure freezing: equipment- and specimen-loading methods. Methods Mol Biol 369:143-173

17. Kurz M, Blattmann B, Kaech A et al (2012) High-throughput counter-diffusion capillary crystallization and in situ diffraction using high-pressure freezing in protein crystallography. J Appl Crystallogr 45:999-1008

18. Otegui MS (2011) The plant cell wall, methods and protocols. Methods Mol Biol 715: 123-140

19. Sobottka B, Ziegler U, Kaech A et al (2011) CNS live imaging reveals a new mechanism of myelination: the liquid croissant model. Glia 59:1841-1849

20. Giddings TH, Meehl JB, Pearson CG et al (2010) Electron tomography and immunolabeling of Tetrahymena thermophila basal bodies. Methods Cell Biol 96:117-141

21. Kaneda M, Rensing K, Samuels L (2010) Secondary cell wall deposition in developing secondary xylem of poplar. J Integr Plant Biol $52: 234-243$

22. Hohenberg $H$, Mannweiler $K$, Mueller $M$ (1994) High-pressure freezing of cell suspensions in cellulose capillary tubes. J Microsc (Oxford) 175:34-43 
23. Monaghan P, Cook H, Hawes P et al (2003) High-pressure freezing in the study of animal pathogens. J Microsc (Oxford) 212: 62-70

24. Schwarb P (1990) Morphologische Grundlagen zur Zell-Zell Interaktion bei adulten Herzmuskelzellen in Kultur. Thesis, Federal Institute of Technology, Zurich, Switzerland

25. Wild P, Schraner EM, Cantieni D et al (2002) The significance of the Golgi complex in envelopment of bovine herpesvirus $\mathrm{l}$ (BHV-1) as revealed by cryobased electron microscopy. Micron 33:327-337

26. Wild P, Senn C, Manera CL et al (2009) Exploring the nuclear envelope of herpes simplex virus 1-infected cells by high-resolution microscopy. J Virol 83:408-419
27. Inamura $M$, Itakura $M$, Okamoto $H$ et al (2006) Differential localization and regulation of stargazin-like protein, gamma- 8 and stargazin in the plasma membrane of hippocampal and cortical neurons. Neurosci Res 55:45-53

28. Michel M, Hillmann T, Mueller M (1991) Cryosectioning of plant material frozen at high pressure. J Microsc (Oxford) 163:3-18

29. Sosinsky GE, Crum J, Jones YZ et al (2008) The combination of chemical fixation procedures with high pressure freezing and freeze substitution preserves highly labile tissue ultrastructure for electron tomography applications. J Struct Biol 161:1-13

30. Möbius W (2009) Cryopreparation of biological specimens for immunoelectron microscopy. Ann Anat 191:231-247 